\title{
Leopardi e Shelley: appunti per un'analisi contrastiva*
}

Per quanto l'ipotesi di un confronto fra la poesia di Shelley e quella di Leopardi possa apparire azzardata, il numero degli studiosi che, fin dalla prima metà dell'Ottocento, l'hanno piú o meno implicitamente suggerita è sorprendentemente alto. Il paragone è stato spesso condotto con modi oggi inaccettabili, su basi generiche o illecite. E non sono mancati coloro i quaii hanno sottolineato, accanto alle plausibili analogie, le insormontabili differenze.

Non si può tuttavia negare, alla luce di alcuni studi recenti che, nonostante tutto, sia rimasto lo spazio per una analisi contrastiva con cui, senza minimizzare le differenze o sopravvalutare somiglianze tematiche e di tono, dovute al clima del periodo, individuare parallelismi concreti e documentati. ${ }^{1}$

All'inizio di un volumetto quasi dimenticato, eppure niente affatto trascurabile, ${ }^{2}$ Guido Biagi inserisce un paragone fra Shelley e Leopardi, solo apparentemente casuale. Accennando al destino di "morte per acqua" che gravò sul poeta inglese fin dalla giovinezza, egli nota che

l'acqua ebbe sempre per lui un'attrazione fascinatrice: egli poteva far suo, ma nel senso proprio, il verso d'un altro poeta sconsolato, del Leopardi: ed annegar $m^{\prime}$ e dolce in questo mare (5). ${ }^{3}$

L'errore nella citazione dell'ultimo verso dell'Infinito ("E il naufragar m'è dolce in questo mare," come si sa) sarà piú un sintomatico lapsus che ignoranza (il verbo annegare infatti compare nel verso precedente: "tra questa/Immensità s'annega il pensier mio").

La coppia verbale, anche se non esattamente sinonimica, amplifica, nel lettore, la portata dell'immagine. Se il poeta si identifica con un'imbarcazione (preferibilmente a vela: indimenticabili, sullo sfondo, le stupende immagini dantesche: "la navicella del mio ingegno" e il "legno che cantando varca") annegare e naufragare coincidono. Shelley parla spesso di "my spirit's bark" (finale di "Adonais"); in "Lines written among the Euganean Hills". ha "my bark, by soft winds piloted. ..." Doppiamente adatta dunque l'epigrafe sulla sua tomba, tratta da The Tempest, I. ii. vv. 397-99 ("Nothing of him that doth fade,/But doth suffer a seachange/Into something rich and strange"), che abbina il fascino della morte per acqua all'altra costante 
su cui ci soffermeremo, quella della "trasformazione," che sembra risolvere la morte in una nuova vita (mito della fenice).

Un'analisi della vita di Shelley basata sui metodi della psicologia moderna ha buon gioco nel dimostrare, come implicitamente ritiene il Biagi, che la fine di Shelley fra le onde del Tirreno equivale a un suicidio: una morte per acqua corteggiata con sottile masochismo, e strenuamente desiderata come autopunizione, dal momento del suicidio della prima moglie, Harriet, nelle acque della Serpentine. ${ }^{4}$ La tentazione è dapprima vagheggiata come uno dei piú dolci modi di annullarsi nella natura, o come resa del soggetto all'irresponsabile, fluttuante gioia dell'utero materno (è facile riconoscere in Shelley l'archetipo del puer aeternus); ̀̀ sublimata talora nella scrittura poetica, come metafora di un amor panico portato all'estrema conseguenza dell'annullamento dell'io poetante.

Per Leopardi si tratta di un sentimento di proiezione nostalgico, forse realmente provato, ma di cui abbiamo traccia retrospettivamente, per esempio nel celebre passo delle "Ricordanze": "lungamente/Mi sedetti colà sulla fontana/Pensoso di cessar dentro quell'acque/La speme e il dolor mio." Ma vari passi dello Zibaldone testimoniano che per la vigile, lucida coscienza leopardiana, il suicidio è impossibile, sia a livello teorico che pratico; esso porterebbe a un istintivo desiderio di sopravvivenza; dall'impulso irrazionale deriverebbe un altrettanto irrazionale amore-sia pur fugace-per la vita:

S'io mi gittassi qui dentro, immediatamente venuto a galla, mi arrampicherei sopra quest'orlo, e sforzatomi di venir fuori dopo aver temuto assai di perdere questa vita, ritornato illeso, proverei qualche istante di contento per essermi salvato, e d'affetto a questa vita che ora tanto disprezzo, e che allora mi parrebbe pregevole.

Per Shelley è quasi un'ossessione. In un atteggiamento antifobico, con củi tenta di distruggere in sé la paura dell'acqua, o di vincere il desiderio di naufragio, il poeta insiste nel descrivere il felice salpare verso oceani azzurri, e navi ansiose di muovere verso l'alto mare, anche se sa, fin dall'inizio, che potrà essere sorpreso dalla bufera;' ma solo i vili non osano, "the trembling throng/Whose sails were never to the tempest given," nel citato finale di "Adonais" (489-90).

Lungi dall'imparare a nuotare, Shelley s'imbarcava sul lago di Ginevra, in compagnia di Byron, con dei pesi addosso, per essere piú sicuro di affondare subito, in caso di incidente, senza mettere in pericolo la vita dell'amico, che avrebbe corso gravi rischi nel tentativo di salvarlo. L'ammirazione di Shelley per Byron, che attraversò l'Ellesponto senza soccombere, come Leandro, si esprime in un linguaggio che proietta l'episodio su un piano mitico, sfiorando tuttavia il ridicolo. L'episodio è demitizzato da 
Byron preventivamente, nell' anticlimax del celebre distico conclusivo di "Written after swimming from Sestos to Abydos": "He [Leandro] lost his labour, I my jest;/for he was drown'd, and I have the ague."

In un delicato schizzo a penna su un manoscritto della Bodleian Library, Oxford, Shelley disegna un lago (o mare) calmissimo, con aguzzi picchi di montagna, sullo sfondo. A destra è un'incantevole isoletta, con una vegetazione eterogenea; sullo sfondo, un'esotica cupola di moschea, con mezzaluna, e un esile minareto. A sinistra una leggera barca a vela, con unico passeggero, scivola verso la meta ideale dell'isola; ma al centro del foglio un altro vascello vola verso il cielo, da dove alcune paia di occhi guardano enigmaticamente. Il tutto, nella struttura, ricorda un' allegoria di Bellini; all'analisi psicologica rivela la tentazione del "folle volo" verso una realtà aerea, rarefatta, un'altra dimensione. Ma se il volo è precluso ad un vascello che non sia fantasma, per evadere resta la soluzione del naufragio. E d'altronde l'idea che la realtà riflessa nell'acqua sia in genere piú bella è, per Shelley, un motivo ricorrente; ne cogliamo gli echi, per esempio, in una pagina del Notebook, del 1821: "Why is the reflection in that canal far more beautiful than the objects it reflects?" (King-Hele 33637). Nel seguito del volume citato, il Biagi esagera nel leggere citazioni leopardiane persino nella descrizione di Mary Shelley che a Pisa chiede a Byron notizie del marito: ". . . ed ella era di pietra, come la donna cantata dal Leopardi, che sola cammina incontro al vento e alla tempesta" (Biagi 40; si veda anche Jonard). Resta il fatto ch'egli testimonia l'evidente impossibilità, per la critica italiana fra la fine dell'Ottocento e i primi del Novecento, di parlare di Shelley senza pensare, per analogia o contrasto, a Leopardi. Non diversamente, come vedremo, sarà a lungo impossibile alla critica inglese evitare, nel discorso su Leopardi, il confronto con Shelley.

La fine dell'Ottocento e i primi anni del nostro secolo erano il periodo della massima fama di Shelley in ltalia (merito del Carducci e del neo-idealismo) e della massima voga del leopardismo, contro il quale, con altrettanta veemenza che contro il manzonismo, si scagliava, appunto, Carducci:

Non lasciarti anche tu rapire al fanatismo leopardiano. è una moda borghese. Cotesta brutta canaglia fa ora col Leopardi come già col Byron. Tutti gli avvocatuzzi, e mediconzoli, gli uffizialetti, e la ragazzaglia, prole di sarti, sente il dolore mondiale, lo scavante nullismo del Leopardi. ${ }^{5}$

Carducci stesso, nel descrivere l'età di Leopardi, la definiva come la piú veramente lirica nella storia della letteratura europea, medioevo a parte, e citava a conferma, oltre a Leopardi, Byron, Lamartine, Hugo, Heine, von Platen, Manzoni e, naturalmente, Shelley (Stufferi Malmignati 141). Ma 
Carducci è una felice eccezione nel contesto del XIX secolo, nel quale i confronti fra poeti venivano basati, di regola, su parallelismi di contenuto, soprattutto ideologico; errore imperdonabile, come sappiamo, ma anche tentazione assai seducente, nel caso di due "poeti filosof" come Shelley e Leopardi. $^{6}$

Infatti, a parte l'acuto articolo apparso anonimo su The Cornhill Magazine nel 1876, secondo cui Leopardi "è melodioso quanto Shelley, serio quanto Wordsworth, impetuoso quanto Byron"7 (l'affermazione è confortata da una discreta traduzione di un brano delle "Ricordanze"), sia Gladstone che Sainte-Beuve, solitamente preziosi anche per dei giudizi limitati a pochi accenni, avevano paragonato i due poeti piú sul piano delle idee che della scrittura poetica, per quanto Gladstone insistesse, un po' ambiguamente, sull' "appassionata malinconia" di entrambi (Stufferi Malmignati 25, 32; cfr. Sainte Beuve). Nell'edizione del 1882 dell' Encyclopedia Britannica Richard Garnett, autore della voce "Leopardi," accostava, non del tutto arbitrariamente, "La Ginestra" a "Mont Blanc," e "L'appressamento della morte" a "The Triumph of Life"; concludeva purtroppo con l'affermazione, che tanta fortuna avrà in Inghilterra nei decenni successivi, che Shelley è superiore a Leopardi perché portatore di una filosofla piú ottimistica. Nella History of Italian Literature (1898) Garnett lasciava da parte, per fortuna, la ricerca del primato e si limitava ad affermare che Shelley e Leopardi erano entrambi "greci"; il primo per il suo panteismo, il secondo per la perfezione, intendendo, con tutta probabilità, non a torto, che l'ellenismo di Shelley è un ideale etico (e in questo il poeta inglese è piú rinascimentale che romantico) (Langbaum 34), mentre quello del maturo Leopardi (come di Keats) è un modello estetico-formale.

Nello stesso 1898 (centenario della nascita di Leopardi) W. Knox Johnson paragonava, su The Fortnightly Review la varietà e la "perfezione" dello stile di Leopardi, a quello di Milton, Shelley e Tennyson. Leopardi è, per Knox Johnson, pari a Shelley per la bellezza immaginativa e la forza irresistibile del canto; lo supera per un maggiore equilibrio, per una maggiore ricchezza. Contro il paragone fra Leopardi e Shelley scriveva intanto, in America, William James; per lui Leopardi non appartiene alla stirpe di Hugo, Hölderlin, Schiller, Keats e Shelley, perché non invoca mai il sentimento. Il suo canto nasce da una solitudine stoica e aristocratica, piú illuminista che romantica. $^{8}$

Mentre in Inghilterra e in America la statura di Leopardi a confronto di Shelley era in ascesa, in ltalia Arturo Graf lamentava la mancanza, nel poeta italiano, di quel "decorativismo paesaggistico" di cui sarebbero ricchi invece Chateaubriand, Byron e Shelley (Stufferi Malmignati 146). 
Leopardi e Shelley sarebbero stati comunque avvicinabili, piú che sul piano stilistico, sul piano della poetica; per entrambi la poesia, in quanto produzione di bellezza, era "contemplation of the Beautiful"; con ciò il Graf non solo sopravvalutava il platonismo di Shelley, ${ }^{9}$ ma attribuiva a Leopardi un idealismo di genere ben diverso da quello che è pur riscontrabile nei suoi versi. ${ }^{10}$ All'inizio del nostro secolo Sir Theodore Martin, nell'introduzione alla sua traduzione (Poems of G. Leopardi) riecheggiava il confronto nei termini proposti dal Garnett, dimenticando, come il Garnett, che per quanto riguarda "The Triumph of Life," il pessimismo che ne ricava il lettore è dovuto al fatto che si tratta di un'opera incompiuta, la cui conclusione, sulla base delle premesse, è lecito immaginare di ben altro tono. ${ }^{11}$

Un progresso solo apparente è nel metodo comparatistico di C.H. Herford il quale, in Shakespeare's Treatment of Love and Marriage (London, 1921) mette a confronto le "filosofie" di Omero, Eschilo, Lucrezio, Dante, Shakespeare, Shelley e Leopardi. Questi ultimi, secondo Herford, idealisti e visionari, sarebbero assimilabili perché vivono della gioia esaltante della loro poesia, in un eterno culto della bellezza; li salva dal pessimismo la fede nell'energia creatrice del pensiero e nell'amore, le due forze che riscattano l'uomo dalla congenita condizione di impotenza.

Il passo avanti piú notevole si compie nel Leopardi di Iris Origo, ricco di spunti di grande finezza, di cui citeremo un solo esempio. Nell'osservare che Leopardi avrebbe potuto incontrare Shelley, Byron e Leigh Hunt a Pisa, la scrittrice si diverte a immaginare l'effetto che la stravagante comunità inglese avrebbe suscitato sul poeta italiano, provinciale schivo, ma già in possesso di un equilibrio di prim'ordine. La conclusione, che gli eccessi nel comportamento di tali personaggi, come dei romantici tedeschi, lo avrebbero piuttosto allontanato che accostato alla loro produzione poetica, è confermata da una serie di notazioni nelle Lettere e nello Zibaldone. Dei tedeschi Leopardi scriveva che

rivelano sentimenti e idee cosí bizzarri, mistici e visionari, che non posso dire che mi piacciano veramente molto. ...

(si osservi l'uso prudente, e ambiguo, dell'eufemismo). Da un lato continuava a lamentarsi che il contatto con le letterature straniere fosse reso arduo dalla difficoltà di procurarsi i testi, in originale o in traduzione:

i moderni qua non arrivano, e io presentemente leggendo sempre, sto in una totale ignoranza delle cose del mondo letterario. ${ }^{12}$

Dall'altro rifiutava le mode che penetravano ogni anno piú sottilmente: 
Nutriamoci d'Ossian e d'altri poeti settentrionali, e poi scriviamo, se siam da tanto, come piú ci va a grado, senza usare le loro immagini e le loro frasi. ${ }^{13}$

Al livro di Iris Origo è premessa un'eccellente introduzione di George Santayana, nella quale si coglie la conciliazione fra quanti insistevano sul pensiero leopardiano (soprattutto in Inghilterra) e quanti cercavano esclusivamente il poeta lirico (in Italia, per influsso di Croce). Per Santayana lo stile di Leopardi è efficace perché sostenuto da un'ispirazione intellettuale di grande profondità e rigore: "la musica è musica di pensieri." Rispondendo idealmente a chi lamentava il carico di pessimismo che gravava sulla sua poesia, Santayana scrive:

La visione di Leopardi era satura di angoscia, limitata da essa, ma non distorta. ... Al di sotto della gloriosa monotonia delle stelle egli vide l'universale mutarsi di ogni cosa terrena, la vanità di tutto, ma insieme, ovunque, l'inizio almeno, se non il compimento della bellezza.

Questo giudizio ci sostiene nell'opinione che, fra le liriche di Shelley, 1" Hymn to Intellectual Beauty" abbia il maggior numero di movimenti leopardiani. E accostabile a Leopardi l'idea che il mistero aggiunge fascino alle cose, piú della loro stessa grazia ("like aught that for its grace may be/Dear, and yet dearer for its mystery"), la definizione della terra come "this dim vast vale of tears," e della condizione umana come "state . . . vacant and desolate"; il riconoscimento amaro che tre cose principalmente governano il mondo, "doubt, chance, and mutability," l'angoscia che alla fine della vita ci aspetti "the grave . . . / Like life and fear, a dark reality" (e per tutta la vita "fear and dream and death and birth/Cast on the daylight of this earth/Such gloom"). Piú che mai leopardiano ("Le ricordanze") ma anche (perché no?) jamesiano, l'accenno al fanciullo che percorre grandi stanze, nutrendosi di visioni, immagini, paure piú grandi di lui: "While yet a boy I sought for ghosts, and sped/Through many a listening chamber"; e l'esaltazione della bellezza intellettuale che, sola, "gives grace and truth to life's unquiet dream" (si noti l'equazione vita/sogno e la bella qualificazione, (unquiet); piú che leopardiana, l'identificazione graceltruth prelude a Keats, nella celebre conclusione dell" "Ode on a Graecian Urn," e a Emily Dickinson ("I died for beauty"). Ancora leopardiano, infine, il canto della giovinezza come primavera della vita, ma stagione tutt'altro che spensierata, anzi periodo in cui sorgono i primi gravi dubbi alla coscienza e l'io oscilla fra il desiderio di abbandono al fascino del momento, prima che fugga, e la riflessione sul destino umano: "musing deeply on the lot/Of life, at that sweet time when winds are wooing/All vital things that wake to bring/News of the birds and blossoming."

Ma per concludere la rassegna della fortuna del confronto fra i due 
poeti, citiamo gli interventi piú moderni. Per l'anonimo autore di un articolo sul Times Literary Supplement (19-6-1937 e segg.) Leopardi è piú "puro" di Shelley; è la voce dell'eterna giovinezza, se giovinezza è una categoria dello spirito. La giovinezza di Shelley, al contrario, è uno stato psicologico ibrido, quasi patologico, di un'adolescenza che non vuole maturare. Per questo, come ben scrive Nesca A. Robb, l'inesausto amore per la libertà di Shelley è assimilabile solo al Leopardi della "Canzone all'Italia." Leopardi sarà anche in seguito il cantore della libertà, ma in termini di precisa consapevolezza intellettuale. La libertà di Shelley è la leggerezza vaga della nuvola, eternamente in movimento, sempre diversa, ma sempre sull'orlo dell'evaporazione (Hall).

L'anelito del poeta a sciogliersi dai vincoli delle convenzioni, del conformismo, trova in Leopardi un'emotività controllata; e il senso di impotenza, lungi dal produrre sterile ribellismo, sfiora il sublime del poeta greco, quando si confronta con anánke, la necessità, il fato. ${ }^{14} \mathrm{La}$ malinconia di Shelley, il sentimento di dejection, è un umore talora accettabile anche a livello artistico, ma piú spesso insistito, quasi patetico.

Il confronto fra la libertà cui anela Shelley e quella che sostiene la poesia di Leopardi si fa legittimo, per il Donadoni, nei casi, non infrequenti in entrambi, in cui essa esprime il sentimento dell'infinito come "dolcezza spirituale e passione cosmica e condizione del filosofico meditare."15 Nicolas James Perella concede anche a Shelley il dono di esprimere a pieno, seppur a sprazzi, l'esperienza del sublime. La dolcezza di atmosfere leopardiane, soprattutto di certi paesaggi lunari, trova il suo equivalente in inglese non solo, per esempio, nell' "Ode to a Nightingale" di Keats, ma nell'altrettanto prezioso quarto atto di Prometheus Unbound, cui appartiene il distico famoso: "Oh gentle moon, the voice of thy delight/falls on me like thy clear and tender light" (4.495-96; cfr. Perella 4). Molto acuto, sempre in Perella, l'accostamento fra la poetica di Foscolo, di Leopardi e di Shelley. Si confronti, in particolare, Zibaldone 1182-83:

l'immaginazione vede il mondo come non è, si fabbrica un mondo che non è, finge, inventa, non imita . . .

con l'epigrafe di Emilia Viviani a "Epypsichidion":

L'anima amante si slancia fuori del creato, e si crea nell'infinito un Mondo tutto per essa, diverso assai da questo oscuro e pauroso baratro. ...

come si vede, è sufficiente porre, come legittimamente si può fare, l'equivalenza fra i due soggetti (l'immaginazione del Leopardi e l'anima amante, da cui scaturisce l'ispirazione poetica) perché le due asserzioni risultino del tutto corrispondenti, a parte la grande distanza nel tono. Stephen Rogers 
studia l'ellenismo e il significato della grecità in Shelley e in Leopardi (e in André Chénier) superando il tradizionale discorso delle fonti comuni e la generica conclusione che i due poeti rappresentano ed esprimono lo stesso clima culturale e un gusto analogo (Rogers). Si sa che Shelley studiò con cura i classici greci a piú riprese, soprattutto nel periodo 18131815 e dopo il 1820); si sa ch'egli non limitò l'interesse unicamente agli autori del Vl-V secolo (i tragici, i lirici, i filosofi pre-socratici, gli storici) o ai poemi omerici; la sua sete di cultura greca lo condusse fino alle opere degli scrittori alessandrini (Polibio, Teocrito, Apollonio Rodio, Teofrasto). Lo stesso si può dire di Leopardi, per il quale la cultura greca fu sempre, comunque, qualcosa di piú naturalmente legato alle proprie radici. Significativa è la predilezione di entrambi per due poeti della tarda grecità, Mosco e Bione. Ma il parallelismo fra i due autori moderni non risiede nella coincidenza dell'imitazione (prima parte di “Adonais”), bensí, molto piú sintomaticamente, nella finale distanza dalla fonte comune, usata solo come spunto per una poesia di grande originalità. Si veda la seconda, piú bella parte di "Adonais," dalla strofe XXXVIII in poi, in cui il modello greco continua a funzionare a livello stilistico (il poeta moderno gareggia con la ricchezza e la sensualità delle immagini, che non cessano di offrire esempi di un linguaggio ricco e artisticamente elaborato, fino al limite di una raffinatezza barocca) ma non piú a livello ideologico e contenutistico.

Per Leopardi e Shelley la Grecia non fu solo letteratura ("la letteratura greca è la piú bella che il mondo abbia mai creato," On The Revival of Literature, 1815), ma l'antica e sempre giovane madre di ogni bellezza. Nella grecità Leopardi e Shelley trovano un ideale di eroismo polifico ("Canzone all'ltalia," 1818, "Hellas," 1821). ${ }^{16}$ Ma la Grecia è anche, per Shelley, armonia perfetta fra sensualità, raffinatezza e razionalità, arte del lògos (non per caso egli tradusse, fra i dialoghi di Platone, il piú sensuale, il Convito); la Grecia di Leopardi è una lezione di stile; il Platone che sorride al di sotto della prosa squisita delle Operette morali è il piú "loico."17

La Grecia è un mito, ed è la fonte dei piú bei miti; i nostri poeti li usano non come ornamento, ma come modelli universali di contenuti eterni. Attraverso l'uso dei miti della tradizione classica leggiamo in forma d'arte gioie e sofferenze della loro complessa personalità, ma anche vediamo rappresentati dolori e speranze che appartengono all'umanità intera.

Riprendiamo, fra tutti, il mito di Narciso, cui abbiamo fatto riferimento per il discorso della "death by water." A dispetto del sincero, quasi masochistico altruismo, Shelley, in quanto, come dicevamo, puer aeternus e artista innamorato solo di se stesso, fu poeta narcisista quant'altri mai. Il mito unisce alla fascinazione della morte per acqua l'idea dell'annullamento nell'attimo del congiungimento con la propria, desiderata immagine spe- 
culare, e l'altra idea, della metamorfosi in fiore, come consolazione e superamento della morte. Di questo mito si possono cogliere infiniti esempi nella poesia di Shelley; piú scoperti quelli della produzione giovanile. In "Alastor" il giovane eroe, nella sua solitudine dedicata agli studi, è una prefigurazione del Leopardi delle "sudate carte"; in piú ha un paio di "wild eyes" e una dose assai piú che leopardiana di narcisismo. Assai simile a questo di "Alastor" il protagonista del romanzo autobiografico che Leopardi progettò, sulle orme del René di Chateaubriand; secondo gli appunti, un giovane d'altissimo ingegno, amante della solitudine e dello studio, ma in piú invaso dall'accidia, dalla noia, dal languore. Nell' "Ultimo canto di Saffo" la poetessa esprime affetti shelleyani (e il movimento, il ritmo del verso giunge a un'inconsapevole mimesi di testi classici di Shelley, come l' "Ode to the West Wind"), in un sentimento panico fatto di odi et amo; corteggia e odia l'acqua che l'attrae e la sfugge, cerca l'oblio dell'affanno nelle tempeste, la consolazione ne "l'etra liquido" nel "flutto/Polveroso de' Noti" (si osservi che non solo il fiume, ma tutto il paesaggio si carica di connotazioni acquee); se non le è concesso di immergersi nell'amoroso, fresco abbraccio del ruscello, potrà "Natar ... tra' nembi." Il senso di esclusione della felicità della natura (in particolare dall'acqua come utero materno) la porterà al tuffo nelle onde dello Ionio dalla rupe di Leucade, in un disperato accesso di narcisismo non corrisposto (né si trascuri di riflettere sulla connessione fra narcisismo e omosessualità, fra complesso materno e non accettazione di sé).

Funzionale in Shelley, non meno che in Leopardi, l'apporto del Tasso. La Gerusalemme fu il primo capolavoro italiano che Shelley poté gustare nella nostra lingua; percorrendo a ritroso la storia della letteratura, passò all'Orlando Furioso, al Canzoniere petrarchesco, giungendo a Dante, di cui le tracce piú scoperte, e piú efficaci, sono in "The Triumph of Life." Ma il Tasso restò, per la sua particolare, ipersensibile personalità, per la moderna e "liquida" musicalità di certe sue ottave, e per quanto di lui era passato nella tradizione inglese del Seicento (Milton soprattutto), il grande amore. Milton è, di contro, una frequente citazione nelle prose di Leopardi.

Come la cultura italiana in Shelley, quella inglese entra a far parte del bagaglio di Leopardi spesso per vie indirette, attraverso traduzioni francesi; giungono cosí a Leopardi la sapienza orientale, i resoconti di viaggi in paesi esotici, le descrizioni di costumi di popoli lontani; i Kirghisi del "Canto notturno," gli Indiani della California dell' "Inno ai patriarchi," i cannibali sudamericani di Popaian ("La scommessa di Prometeo," nelle Operette morali).

In Leopardi, come in Shelley, è evidente l'eredità dell' empirismo, non 
mai del tutto cancellata dal gusto dell'età romantica: Butler, Berkeley, e Shaftesbury inducono l'interesse costante per l'etica del sentimento; Addison, Hobbes, Burke, Locke, Hume offrono l'esempio di un'attenzione insostituibile al fatto, al dato materiale, all'esperienza, nei cui confronti la proiezione metafisica e mistica è a lungo vissuta come tradimento. Offrono anche il modello di una prosa urbana, raffinata, fluida, antiretorica per eccellenza. Si confrontino, per le soluzioni formali e il tono, in perfetto equilibrio fra humour e serietà di intenti, le Operette morali con On the Devil and Devils, piccolo capolavoro di Shelley prosatore. ${ }^{18}$

Detto questo, apparirà molto meno ambiguo e sconcertante quel black humour che fa del "Dialogo di Federigo Ruysch e delle sue mummie" una delle piú moderne creazioni leopardiane, condotto sul filo di quel mélange fra macabro e ironico, che consideriamo una provincia esclusiva della letteratura inglese. Non siamo d'accordo, quindi, con le riserve espresse sul dialogo da uno studioso pur attento ai rapporti di Leopardi con le letterature moderne, come Domenico Consoli (n. 21).

La tensione dialettica fra empirismo, scientismo, e spiritualismo, idealismo, conduce Shelley sull'orlo di uno sdoppiamento della personalità, che si traduce in un manicheismo piú subíto che accettato. Ma dopo il crollo di Zeus e il trionfo di Prometeo, tale dualismo si risolve nel panteismo, nella religione dell'Uno, nella dottrina della solidarietà, cui si accosta la conclusione della "Ginestra." La natura, la scienza, in Shelley, non sono mai, di per sé, "cattive"; sono le istituzioni, corrotte, all'origine dell'infelicità umana ("Queen Mab"). ${ }^{19}$

La consolazione non giunge per via razionale. Sia Shelley che Leopardi hanno fede nell'Assoluto. Shelley crede nell'immortalità dell'anima e oscilla fra il desiderio di un Eden terreno e il sogno di una vita ulteriore. Leopardi nega la vita futura, ma non è scettico come Byron, l'unico poeta romantico veramente nichilista. L'Assoluto di Leopardi è l'ansia, non la certezza dell'ideale, l'espressione, non il possesso della bellezza.

Nel contesto, la poesia è, per entrambi, la maggiore forza ristoratrice, la vittoria contro dubbi, paure, senso di impotenza; la soluzione all'esilio in questa "valle di lacrime." In A Defence of Poetry, come osservava il Croce, Shelley "esalta la poesia sopra se stessa," il che non accade mai in Leopardi. Il poeta inglese sopravvaluta il valore e il potere dell'immaginazione e dell'ispirazione nei confronti della riflessione e della revisione, in contrasto, questa volta, non solo con Leopardi (si veda lo sdegno, implicito nella conclusione dello "Scherzo": "Musa, la lima ov'è?/Disse la Dea:/La lima è consumata, or facciam senza") ${ }^{20}$ ma con tutta la poesia moderna, da Poe e Baudelaire, dai parnassiani a Eliot e Pound.

L'ideologia shelleyana trova il punto di maggiore coesione emotiva 
nel mito di Prometeo, che vive del contrasto fra senso di impotenza e desiderio di rivolta contro l'ingiustizia. I punti di contatto con Leopardi sono, in questo caso, facilmente dimostrabili. Fra l'altro, non è un caso che la prima, e a lungo l'unica poesia di Leopardi tradotta in inglese sia stata proprio la "Canzone all'Italia." ${ }^{21}$ Non intendiamo ripetere concetti esaurientemente analizzati da Umberto Bosco, ma l'accostamento dell'interpretazione leopardiana con quella shelleyana non cessa di gettare nuova luce sull'uno e sull'altra personalità poetica (Bosco).

In Leopardi la ribellione di Prometeo è rivolta contro una onnipotente figura paterna; l'identificazione del padre, per via del bigottismo di Monaldo, col "sommo Giove" cattolico, gli impedisce di sfociare nell'identificazione di Prometeo con Cristo tramite la dottrina del perdono, che è la geniale soluzione di Shelley. L'interesse per il mito di Prometeo e dei Titani è documentato in Leopardi dalla versione della Titanomachia e da memorabili pagine autobiografiche in cui si esprime in grida laceranti il presentimento della morte e della conseguente rinuncia alla lotta, alla gloria, al potere; e infine, dalle note intorno a un progettato inno ad Arimane, il dio del male. ${ }^{22}$

Il piacere della propria salute e bellezza, anche se non garantisce la vittoria finale, fa accettare all'io poetante di Shelley il destino di frustrazione che attende gli animi piú nobili; si pensi all'autoritratto di "Adonais" (vv. 280-82): "A pard-like spirit beaufiful and swift-/A love in desolation masked;--a Power/Girt round with weakness." Leopardi resiste al narcisismo, contempla il proprio velleitarismo giovanile dall'esterno e lo trova non già sublime, ma ridicolo, patetico. ${ }^{23}$

Per quanto Leopardi sia piú radicalmente pessimista di Shelley, la dejection di quest'ultimo è uno stato mentale non meno frequente della noia leopardiana; entrambe utilissime a livello artistico, in quanto spesso la produzione poetica è proprio una reazione al "tedium, boredom, unfocussed dissatisfacfion ... the disease of an active mind"; ${ }^{24}$ ma la dejection di Shelley si muta continuamente in depressione e prostrazione, umori nemici dell'ispirazione.

In "The Triumph of Life" la descrizione della vita ha toni piú amari che nel Leopardi piú sconsolato; come in Leopardi, la piú gran pena è l'angoscia della non risposta: perché siamo al mondo? chi, che cosa ci costringe a vivere?; "Se la vita è sventura/Perché da noi si dura?" Shelley suggerisce l'affascinante ipotesi che alcuni coraggiosi abbiano saputo rifiutare la vita nell'istante stesso in cui vennero al mondo, non cedendo a lusinghe di alcun genere; non già il suicidio, ma una sorta di intuizione fulminea, che fa loro conoscere in un istante la vita, prima di "firmare il patto," prima che il velo di Maia li condizioni per sempre; un immediato 
ritorno al di là della soglia dei non nati: "the sacred few who . . . . . . as soon/As they had touched the world with living flame,/Fled back like eagles to their native noon." 25

Leopardi concorda che la vita è male; la morte è la fine della sofferenza; non va affrettata, invocata, come fa il giovane con atteggiamento poco virile, bensí attesa serenamente, oltre ogni sofferenza: "aspetto molto quietamente quello che porterà il tempo." ${ }^{, 26}$ L'approdo di Shelley maturo non sarà mai questa rassegnazione, bensí la scoperta (seconda parte di "Adonais") che la morte è un destarsi alla vera vita, secondo il tòpos della "vita come sogno," "sogno di un'ombra" (Pindaro), "ombra di un sogno" (Carducci).

In attesa della morte, vuoi come annullamento che come indizio di una vita migliore, resta il problema del rapporto con la natura. Shelley oscilla moltissimo nel descrivere il rapporto fra lo stato d'animo dell'io poetante e il paesaggio in cui esso si trova immerso. La natura può essere, nella sua meraviglia, una "compensazione" dello stato di dejection ("Stanzas written in dejection, near Naples"); nella sua apparente tristezza, può consolare, illudendo l'uomo di una partecipazione al suo dolore ("Evening: Ponte al mare, Pisa"); può offrire istanti di intensa emozione e di oblio dell'angoscia ("Lines written among the Euganean Hills"); può rapire il soggetto nel vortice della sua infinita potenza ("Ode to the West Wind"); il piacere sommo è, forse, nei rari attimi di meditazione nella natura, fino all'assopimento progressivo della coscienza, invasa dal silenzio, dalla maestà del paesaggio, dall'armonia, dalla luce, sia essa di un meriggio assolato o di un plenilunio sereno. Leopardi mostra una simile gamma di reazioni, ma sempre temperate dal momento della riflessione "in studio" (per quanto belli, vivi, coloriti, i suoi paesaggi non appartengono ancora all'età del plein air). Sicché, stranamente, è piú Shelley, e altri poeti stranieri, che non Leopardi, a influenzare il paesaggio della poesia italiana del secondo Ottocento e di certo Novecento. L'oblio di Carducci nel meriggio, promesso ed evocato dai cipressi ("Davanti San Guido"), piú che della splendida descrizione meridiana nella "Vita solitaria," è debitore di Shelley; e D'Annunzio deriverà dal secondo romanticismo inglese (anch'esso filiazione di Shelley) e dal simbolismo francese. La "gloria del disteso mezzogiorno" non arricchisce forse la memoria di Leopardi (e Carducci) con la gloria del meriggio sulla pianura veneta, contemplato dai colli Euganei? E il cuore di Montale, che desidera essere suonato dal "vento che nasce e muore/Nell'ora che lenta s'annera" non deriverà dall'ardente auspicio dell" "Ode to the West Wind": "Make me thy lyre"?

Gli esempi di sentimento panico sono innumerevoli in Shelley, numerosi in Leopardi. Confessa Bacchelli di preferire Leopardi, per "una sfumatura 
e un'inflessione di tono meno arduo, meno severo . . piú familiare e meno sublime" (148).

Inscindibile dal sentimento panico è, nei due poeti, il concetto di infinito, a sua volta collegato, come si sa, al concetto di sublime, per il quale sarà stata fonte comune il pensiero di Burke. Shelley si discosta alquanto da Leopardi, in questo, perché manca in lui quell'istante di suspence, quell'attimo di paura prima del tuffo, dell'abbandono ("ove per poco/Il cor non si spaura"). Il "delightful horror" di Burke è, per Shelley, affascinante fin da principio, senza residui. ${ }^{27}$

Shelley concorda col noto saggio apparso sullo Spectator del 1712 , tradotto in francese e citato da Leopardi nello Zibaldone:

alla mente dispiace tutto ciò che la costringe e gode quando non ci sono prigioni né confini: donde il senso del sublime e del grandioso. . . ${ }^{28}$

Leopardi è piuttosto vicino a Milton e al famoso "muro" di Berkeley; l'infinito si può concepire solo per contrasto con l'idea di finito, e meglio godere se c'è una:

veduta ristretta e confinata in certi modi. . . Perché allora in luogo della vista, lavora l'immaginazione e il fantastico sottentra al reale. L'anima s'immagina quello che non vede. . . . ${ }^{29}$

Sia Leopardi che Shelley sono, come tutti i maggiori poeti moderni, cantori della mutabilità e della trasformazione. Il senso di precarietà del presente porta Shelley ad auspicare la metamorfosi, donde il soggetto riuscirà rinnovato, come la farfalla che ha saputo pazientare nel bozzolo, dotato in piú di una coscienza "pervasiva"; la nuvola è felice perché vola leggera e assume le piú varie forme; il riflesso nel canale, come s'è visto, è piú bello della realtà che riflette, perché non illude della permanenza delle cose; il poeta mutato in foglia potrà essere trascinato dall'alito del vento; mutato in puro strumento, sarà le corde vocali della natura. La capacità infinita di mutare è un altro modo di vincere la morte: "I change, but I cannot die" ("The Cloud").

Se Shelley crede di poter trasformare ogni cosa (la natura, il destino dell'uomo; metamorfosi come attività dell'io, che modifica di continuo il non-io) Leopardi accetta l'idea che tutto è in perenne trasformazione (metamorfosi come stoica passività). Come per Eliot, cosí per Leopardi la primavera è "the cruellest month," non solo perché rinascono i germogli, ma perché tale rinascita illude di un ritorno e quindi di un ciclo perenne.

Il meglio della poesia di entrambi è nei versi in cui il mondo interiore, per quanto fragile e turbato, ipersensibile e gravemente meditativo, aderisce al mondo esterno, allo spettacolo di una natura luminosa, vasta, multi- 
forme, dinamica, armoniosa. In Shelley, piú che in Leopardi, la natura è spesso iper-romantica, turbolenta, selvaggia, pittoresca, alla Salvator Rosa; ma si veda l'abbozzo leopardiano del $5^{\circ}$ "Argomento di Elegia" (non si sa se piú byronico o shelleyano):

Elegia di un innamorato in mezzo a una tempesta che si getta in mezzo ai venti e prende piacere dei pericoli che gli crea il temporale ed egli stesso errando per burroni ecc. ...

Al sogno del pastore errante ("forse s'avessi io l'ale/Da volar su le nubi/E noverar le stelle ad una ad una,/O come il tuono errar di giogo in giogo") risponde in un invito la nuvola di Shelley: "I wield the flail of the lashing hail,/And whiten the green plains under,/And then again I dissolve it in rain,/And laugh as I pass in thunder." E replica Leopardi: "Si rallegra il cor quando a tenzone/Scendono i venti, e quando nembi aduna/L'Olimpo, e fiede le montagne il rombo/Della procella" ("Nelle nozze della sorella Paolina"). Al passero solitario ("D'in su la vetta della torre antica/. . . alla campagna/Cantando vai finché non more il giorno;/Ed erra l'armonia per questa valle") corrisponde l'allodola: "Hail to thee, blithe Spirit!/Bird thou never wert,/That from heaven, or near it,/Pourest thy full heart/In profuse strains of unpremeditated art"; si osservi, al di là delle differenze, l'effetto di dilatazione sonora all'infinito nell'ampio verso conclusivo di queste ultime citazioni. ${ }^{31}$

Uno dei profitti dell'analisi che siamo andati compiendo sarà la conferma che anche due poeti considerati, a buon diritto, diversissimi sotto tanti aspetti, rivelano, accostati "per chiaro-scuro," singolari punti di contatto. Passiamo brevemente in rassegna alcuni paesaggi notturni, nei quali anche Shelley, miracolosamente, coglie la magia della natura con la stessa religiosa astensione dall'intrusione soggettiva (tanto piú difficile per il suo temperamento) e con simile gusto per la "carrellata panoramica" che esprime in termini visivi la dilatazione e l'amplificazione sonora che abbiamo notato nelle citazioni precedenti. Da un punto di partenza preciso l'occhio del poeta muove lentamente, come una.macchina da presa che arretri, per inquadrare una porzione sempre maggiore del panorama, e scorra poi a descriverne la maestosa ampiezza: "E tu pendevi allor su quella selva/Siccome or fai, che tutta la rischiari" ("Alla luna"); "The cold white light of morning, the blue moon/Low in the west, the clear and garish hills,/The distinct valley and the vacant woods" (" Alastor,"19395). La luna è, per Shelley, generalmente "a most enamoured maiden" (si pensi al mito di Endimione e Selene che trovava proprio in quegli anni espressione suprema nel poemetto di Keats), mentre per Leopardi è lontana, nella sua eterna, misteriosa perfezione. Ma non mancano esempi di 
maggiore familiarità con la "solinga, eterna peregrina"; ella può essere, ad esempio, "graziosa," "bella e misericordiosa, gentile" ("Alla luna"). E in Shelley, al contrario, possiamo avere una luna enigmatica, maestosa, irraggiungibile ("Studded with stars unutterably bright,/Through which the moon's unclouded grandeur rools ...," "Queen Mab," 4-6) oppure, come quella del "Canto Notturno," silenziosa, pensosa e muta alle domande del soggetto: "Art thou pale, for weariness/Of climbing heaven, and gazing on the earth,/Wandering companionless/Among the stars that have a different birth,- - And ever changing, like a joyless eye/That finds no object worth its constancy?" ("To the moon"). Si accosti l" "ancor non sei tu paga ... ancor sei vaga/. .." alla "weariness" (implicita, in entrambi i testi, la monotonia del viaggio); il "gazing on the earth" a "contemplando i deserti" e "mirar queste valli"; il "wandering companionless" a "solinga, eterna, peregrina," e si rifletta sul valore storico e poetico di coincidenze cosí significative.

L'ambivalenza delle immagini della luna può essere collegata all'analogo sentimento nei confronti del femminile, le cui figure sono viste da entrambi talora come dolci, gentili, accostabili, talora lontane, perfette, irraggiungibili, o severe, mute di fronte al sentimento, segreto o manifesto, del soggetto poetante. In Shelley prevale la soluzione di proiettare la donna sul piano dell'ideale, anche quando, inizialmente, la persona amata e cantata è una precisa figura umana, come l'Emilia Viviani dell'Epypsichidion; in tali casi la donna regolarmente si muta in quella che Jung chiama $a$ nima, ossia l'immagine archetipica del femminile presente nella psiche del poeta. $^{32}$

In Leopardi non mancano esempi di un atteggiamento simile; si pensi a "quella eccelsa imago" di "Aspasia" (donde l'amore è "smisurato," gli affanni "intensi," i moti dell'animo "indicibili"). Nel vagheggiamento di figure femminili sull'orlo della mitizzazione, che gli impedisce di vedere la donna com'è (tutte le donne sono una, sempre la stessa) Shelley esprime la fuggevolezza del sentimento che prova, l'irrealizzabile sogno, il senso di nostalgia prima ancora che l'amore sia stato appagato. Ma il dialogo non è a senso unico; c'è almeno la speranza della corrispondenza, la finzione, l'ipotesi della felicità d'amore. In Leopardi la figura femminile, per quanto descritta con vibrante partecipazione emotiva, con pennellate indimenticabili, resta oggetto dell'effusione dell'uomo, allontanata, piú che avvicinata, dalla dolcezza del ricordo, o separata dalla morte. Il sorriso di una donna che si posa sul poeta è casuale, inconsapevole, non è coscienza di sentimento e può solo illudere.

In "A Silvia," la donna indica infine la soglia di una tomba. In "Sopra un bassorilievo antico sepolcrale" e "Sopra il ritratto di una bella donna," 
ella è già divenuta immagine marmorea. In Shelley spesso una tomba simbolica attende la donna, il poeta, la speranza d'amore; unica testimonianza, comunque, di un passato che pur è esistito ("Lines, to Harriet"). L'accostamento, ambiguo e affascinante, fra la donna, giovane, bellissima, e la tomba, prelude a Poe (Ulalume).

Sintomatica la differenza nella descrizione di un canto, di una musica prodotta dalla figura femminile; in Shelley prevale l'elemento dionisiaco, la melodia è addirittura un "ritmo selvaggio," accompagnato dall'arpa ("To Sophia"); in Leopardi prevale l'elemento apollineo e il canto, come quello di Silvia, è innocente, virginale, dolce e quasi monotono ("perpetuo") (Anderson). Eppure non mancano singolari coincidenze stilistiche: nella citata lirica di Shelley abbiamo, in efficace contrasto, "deep eyes" e "soft clear fire": gli occhi sono profondi, scuri, pensosi, e lanciano uno sguardo ardente (clear fire) e tenero assieme (soft); gli occhi di Silvia, come ricordiamo, sono "ridenti e fuggitivi," allegri e schivi; negli appunti per la stesura del canto, Leopardi è stato a lungo dubbioso fra "molli," "dolci," "vaghi," "incerti," sempre in contrasto con "ridenti."

Queste osservazioni ci danno modo di concludere con un giudizio piú generale sul procedimento di composizione poetica nei due autori. Leopardi percorre tutti gli spazi possibili alla ricerca dell'unico "mot juste," provando e riprovando, cancellando e sostituendo; alla fine non resta traccia del travaglio, perché non ha temuto di sacrificare ciò che non lo soddisfaceva a pieno. Al contrario Shelley, con piú impeto, ma anche piú approssimazione, aggiunge un verso all'altro, un'immagine icastica ad una piú vaga, senza rinunciare a nulla, in una foga davvero, ripetiamo, dionisiaca, che esprime l'ebbrezza di un'abbondanza, di una generosità, ma anche di un'indulgenza con sé stessi, in un affetto quasi morboso persino nei confronti della propria imperfezione (Keach).

\section{Università di Roma "La Sapienza"}

\section{NOTE}

* 11 Presente saggio prende le mosse da un intervento dell'autore al Seminario di Studi Leopardiani tenutosi a Recanati, settembre 1980 ("Leopardi e le letterature straniere," dir. Prof. A. Russi).

1 Perché il lettore possa intendere da quali basi muove il presente discorso comparatistico, si vedano i contributi teorico-metodologici di E.R. Etiemble, R. Wellek (with A. Warren), R. Wellek, D. Secrétan. Non si deve, a rigore, dimenticare che anche testi piú antichi, per quanto datati, conservano un'efficacia indubitabile; cfr. per esempio A. Béguin, L'âme romantique e Le romantisme allemand. A livello storico si deve 
partire dai celebri interventi di F. Schlegel, di C.A. Sainte-Beuve e di M. Amold. Si tengano presenti infine A.S. Rosenthal e A. Prete.

2 Il Biagi s'era occupato a lungo dell'argomento; già nel 1891 era apparso un articolo a suo nome sul New Harper Magazine di New York, da cui era poi derivato The Last Days of P.B. Shelley.

3 Va detto per inciso che la situazione archetipica della "morte per acqua" (annegamento o naufragio) ha pesato piú gravemente sulla letteratura inglese e americana che su altre letterature occidentali, sia a livello biografico, magari come tentazione, che artistico: va da Ofelia alla finzione della Tempesta, dal marinaio fenicio di Eliot a Virginia Woolf, da Margaret Fuller a An American Tragedy, dal nuotatore impavido di Whitman a Emily Dickinson, da Far from the Madding Crowd al Maelström di Poe, da Moby Dick a E.M. Forster (The other Boat), dall'Open Boat di Stephen Crane al Deutschland di G.M. Hopkins, e cosí via. Il poeta, non solo angloamericano (si pensi a Ippolito Nievo), novello Leandro, non ha sempre trovato, a salvarlo, come Ulisse, Leucotea, o come Arione, il delfino.

4 I biografi ricordano il naufragio di Shelley nella Manica, quando fuggiva sul Continente con Mary Godwin, e il pericolo di naufragio sul lago Lemano, in compagnia di Byron. La ricerca di una morte per acqua si concretizzò in una spirale di sempre piú numerosi naufragi, reali o possibili, nei quali purtroppo, negli ultimi mesi, il poeta rischiava di coinvolgere innocenti compagni di viaggio, fino alla follia dell'ultima avventura. Cfr. K. Folliot, Shelley's e Essays, e C. Tomalin.

5 Lettera a Lidia (1874) in G. Carducci, IX. Per il termine leopardismo concordiamo con le osservazioni di G. Lonardi, Leopardismo, secondo il quale, in senso stretto, non è mai esistito un filone leopardiano nella poesia italiana; dello stesso autore si veda anche "Leopardi " $6-49$, in cui, di passaggio, si definisce la poesia del Carducci delle Odi barbare come il risultato del combinato influsso di Leopardi, Whitman e Wagner; a noi sembra che molto Carducci, non tanto forse quello delle Odi barbare, quanto quello delle raccolte precedenti, derivi anche da Shelley, e da Byron (si pensi al titanismo e al satanismo dell" "Inno a Satana"). Al contrario, è esistito in Inghilterra, e forse ancor piú in America, il filone shelleyano, e una serie di revivals di interesse nel poeta, nella sua vita, nella singolarità della sua esperienza, di cui sono testimonianza le pagine della "Prefazione" di H. James a The Aspern Papers, con l'efficace descrizione di "an American of long ago, an ardent Shelleyite," per il quale le carte di Shelley divengono il fine del corteggiamento dell'anziana Jane Clairmont. Si noti che la Clairmont, in quanto non solo cognata di Shelley, ma compagna di Byron e madre di sua figlia Allegra, avrebbe potuto possedere importanti inediti di Byron stesso, la cui appetibilità, evidentemente, era assai minore: cfr. G. Mclchiori.

6 Si vedano, per la fama di Shelley, non solo nel mondo anglosassone, T. Webb, D. Palmer, e N. Forgarty.

7 Il poeta-filosofo è, per George Santayana, il massimo che si possa dare nella storia della letteratura. Per quanto amasse e avesse tanto studiato sia Shelley che Leopardi, i tre poeti-filosofi per eccellenza erano, per lui, Lucrezio, Dante, Goethe. Si vedano anche R.G. Woodman, D. Bini, O.M. Casale (ed.), M.A. Rigoni, A. Prete, Leopardi Pensieri.

8 Per la prima parte di questo nostro excursus sulla fortuna del confronto Shelley/Leopardi ci siamo basati, naturalmente, sul prezioso Leopardi e l'Inghilterra di C. Singh.

9 Si vedano gli atti dell convegno Leopardi e l'Ottocento, e inoltre A. Vallone, A. 
Valentini, N. Bonifazi, Leopardi Canti.

10 Per un'eccellente discussione del "platonismo" di Shelley si veda E. Chinol, cap. V.

11 Cfr. gli atti del convegno Leopardi e il Novecento, soprattutto i saggi di M. Guglielminetti e M. Petrucciani.

12 Ancora dipende dal Garnett de dal paragone fra "The Triumph of Life" e "l'appressamento della morte" T.J. Farmer. La giustificazione del confronto è, di nuovo, inadeguata.

13 Lettera a P. Giordani, (27-XI-1818), in Lettere LXX1X: 149; cfr. D. Consoli.

14 È la celebre lettera alla Biblioteca italiana (18-Vll-1816) in risposta a Mme de Staël; cfr. Poesie Prose, II. 603. Cfr. Gilardino.

15 M. Cooper avvicina la "sobrietà di linguaggio" in cui si esprime questo sentimento piuttosto al virgiliano "sunt lacrimae rerum."

16 Donadoni, in Leopardi e il Novecento, cit. da Bassotti.

17 Dopo qualche anno, in "To Helen" di Poe, troviamo il verso famoso "the glory that was Greece"; bellezza e gloria sono, nella giovanile sensibilità romantica, inscindibili.

1811 primo autore greco tradotto da Shelley fu Plutarco, che lo interessò, crediamo, soprattutto come fonte di Shakespeare, q. $v$. Webb. Anche per Whitman i Greci saranno maestri di vita e d'arte, di oratoria e di raffinato piacere: "the fluent-tongued and subtle-sensed Greek" in "Song of the Broad-axe." Si vedano anche Carvonara Taddei e Peruzzi.

19 Cfr. Chinol 131. Altre fonti comuni, fra i moderni, C.F. Volney (Les Ruines), The Seasons di Thomson, e Pope, Young (Night Thoughts), Cowper, che variamente confluiscono nello Zibaldone e in "Queen Mab," "The Necessity of Atheism," ecc. Cfr. anche gli atti del convegno Leopardi e il Settecento, in particolare W. Binni e M. Sansone. Per quanto riguarda Godwin (Political justice), cosí importante per Shelley, si deve escluderne la funzionalità dell'apporto, sia pure indiretto, in Leopardi. Certo anarchismo ed esasperato individualismo di Leopardi giovane deriverà piuttosto da Rousseau.

20 Per il pensiero politico di Shelley, cfr. D. King-Hele, Shelley, ma anche il suo Shelley Revalued. Si veda anche T.A. Hoagwood.

21 Per Leopardi la vera poesia è opera di immaginazione, ed è, propriamente, quella greca; la poesia moderna dovrebbe "accontentarsi" di partire dal sentimento (cfr. Zibaldone, 734-35). Cfr. Shelley, La difesa della poesia: Binni, La nuova poetica leopardiana; Rigoni, Stella.

22 Il traduttore, Wraugham, era amico di Wordsworth, Tennyson, Gladstone. (cfr. A Winter's Wreath.)

23 Cfr. Levi. Non riguarda tale tematica la deliziosa "operetta morale" "La scommessa di Prometeo," che affronta la questione della possibilità della civiltà umana in qualche luogo piú o meno remoto del globo. Per Bickersteth è Leopardi, non Shelley, il vero figlio di Prometeo; il poeta italiano è paragonato a Wordsworth (la sua poesia piú wordsworthiana sarebbe "La ginestra"); cfr. anche King-Hele 198. Il titanismo di Leopardi e Shelley giovani deriva anche dal Tasso e da Milton, in Shelley dai drammi elisabettiani dell'ultima fase. Cfr. anche Wasserman.

24 Kroeber 125. Poco oltre l'autore aggiunge ai quattro poeti del titolo Shelley; tutti e cinque sarebbero esempi di poeti romantici che esprimono, talora, sentimenti quasi trascendentalisti, mentre altrove si dimostrano del tutto "non-Swedenborgians or protoBlavatskians" (133). 
25 "The Triumph of Life," 128-31; cfr. Il trionfo della vita e altre poesie.

26 Lettere, 344. 545-46.

27 Si accostino gli ultimi versi dell" "Infinito" con questo distico da "Prometheus Unbound": "Till they fail, as I am faling Dizzy, lost, yet unbewailing." Cfr. anche Leighton.

28 I I piaceri dell' immaginazione, del dott. Akenside (Paris: Mark, 1764); cfr. Zibaldone 706.

29 Cfr. Parronchi. Perella, basa l'analisi del concetto di infinito sul confronto fra "La sera del di di festa" e "The Night Thoughts" di E. Young.

30 Hail, The transforming image, ma anche Sanesi (a cura di), Poesie di P.B. Shelley.

31 La natura è fatta non solo di spettacoli grandiosi, di animi nobili, ma anche di modeste creature notturne; ai timidi rospi che sbucano dai nascondigli ("The swallows are asleep ... . The slow soft toads out of damp corners creep," "Evening: Ponte al mare, Pisa") risponde "il canto/Della rana rimota alla campagna" ("Le ricordanze").

32 King-Hele Shelley, 270 e segg.; Allsup; Brown; Holmes (ed.). Cfr. anche Di Ciaccia.

\section{OPERE CONSULTATE}

AA. VV. Leopardi e l'Ottocento. Firenze: Olschki, 1970.

AA. VV. Leopardi e il Novecento. Fịrenze: Olschki, 1974.

ABERCROMBIE, Lascelles. The Idea of Great Poetry. London: Secker, 1926.

ALLSUP, J.O. The Magic Circle. Port Washington: Kennicat, 1976.

ANDERSON, E. Harmonious Madness: A Study of Musical Metaphors in the Poetry of

Coleridge, Shelley and Keats. Salzburg: U of Salzburg, 1975.

ARNOLD, M. On the Modern Element in Literature. Oxford: 14-XI-1857.

BACCHELLI, R. Leopardi. Milano: Mondadori, 1962.

BASSOTTI, D. La religione di G. Leopardi. Brescia: Morcelliana, 1980.

BÉGUIN, A. L'âme romantique et le rêve. Marseilles: Èditions Cahiers du Sud, 1937.

. Le romantisme allemand. Études publiées sous la direction d'A. Béguin. Paris:

Union Gén. d’Èditions, 1966.

BIAGI, G. Gli ultimi giorni di P.B. Shelley, con nuovi documenti. Firenze: Società

Editrice La Voce, 1922. . The Last Days of P.B. Shelley. London: Unwin, 1898.

BICKERSTETH, G.L. Canti di Leopardi. Cambridge: Cambridge U P, 1923.

BINI, D. A Fragrance from the Desert: Poetry and Philosophy in G. Leopardi. Saratoga, Cal.: Anma Libri, 1983.

BINNI, W. "Leopardi e la poesia del secondo Settecento." AA.VV. Leopardi e il Settecento. Firenze: Olschki, 1964.

. La nuova poetica leopardiana. Firenze: Sansoni, 1984.

BLASUCCI, L. Leopardi e i segnali dell' infinito. Bologna: Il Mulino, 1985.

BONIFAZI, N. Lingua mortale. Genesi della poesia leopardiana. Firenze: Sansoni, 1984.

BOSCO, U. Titanismo e pietd in G. Leopardi. Firenze: Le Monnier, 1957. Ora, con nuovi studi, Roma: Bonacci, 1980.

BROWN, N. Sexuality and Feminism in Shelley. Harvard: Harvard U P, 1979.

CARDUCCI, G. Lettere. Ediz. nazionale. Vol. 9. Bologna: Zanichelli, 1938-1960.

CARVONARA Taddei, M. Momenti del pensiero greco nella problematica leopardiana. 
Lecce: Milella, 1977.

CASALE, O.M., ed. A Leopardi Reader. Urbana: U of Illinois P, 1981.

CHINOL, E. Shelley. Napoli: Edizioni Scientifiche Italiane, 1961.

CIRCEO, E. La poesia satirico-politica di G. Leopardi. Roma: Ateneo \& Bizzarri, 1978.

CONSOLI, D. Cultura, coscienza letteraria e poesia in G. Leopardi. Firenze: Le Mon-

nier, 1967.

COOPER, M. Ideas and Music. London: Barrie \& Rockliff, 1965.

CRONIN, R. Shelley's Poetic Thought. London: Macmillan, 1981.

DI CIACCIA, F. Le donne nella vita di Leopardi e la sua teoria dell'amore. Milano: Nuovi Autori, 1985.

DOTTI, U. Storia di un' anima. Milano: Rizzoli, 1982.

ETIEMBLE, E.R. Comparaison n'est pas raison. Paris: Gallimard, 1963.

FARMER, T.J. The Great Poets of Italy. Toronto: U of Toronto P, 1916.

FOGARTY, N. Shelley in the XXth Century. Salzburg: U of Salzburg, 1976.

FOLLIOT, K. Shelley's Italian Sunset. Richmond: H. \& B., 1979.

. Essays on Shelley. Liverpool: Liverpool U P, 1982.

GILARDINO, S.M. La scuola romantica: la tradizione ossianica nella poesia dell' Alfieri, del Foscolo e di Leopardi. Ravenna: Longo, 1982.

HALL, J. The Transforming Image: A Study of Shelley's Major Poetry. Urbana: U of Illinois P, 1980.

HOAGWOOD, T.A. Prophecy and the Philosophy of Mind: Traditions of Blake and Shelley. University, Ala.: U of Alabama P, 1985.

HODGART, P. A Preface to Shelley. London-New York: Longman, 1985.

HOLMES, R., ed. Shelley on Love: An Anthology. London: Anvil Press, 1980.

JONARD, N. G. Leopardi: essai de biographie intellectuelle. Paris: Les Belles Lettres, 1977.

KEACH, W. Shelley's Style. New York-London: Methuen, 1980.

KING-HELE, Desmond. Shelley: his Thought and Work. 3rd ed. London: Mcmillan, 1984.

Shelley Revalued: Essays from the Gregynog Conference. Leicester: Leicester U P, 1983.

KROEBER, K. The Artifice of Reality: Poetic Style in Wordsworth, Foscolo, Keats and Leopardi. Madison: U of Wisconsin P, 1964.

LANGBAUM, R. The Poetry of Experience: The Dramatic Monologue in Modern Literary Tradition. New York: Norton, 1957.

LEIGHTON, A. Shelley and the Sublime: An Interpretation of the Major Poems. Cambridge, N.Y.: Cambridge U P, 1984.

LEOPARDI, G. Canti. A cura di G. e D. De Robertis. Milano: Mondadori, 1978.

. Canti, a cura di A. Tartaro. Roma-Bari: Laterza, 1984.

. Operette morali, a cura di G. Getto con commento di E. Sanguineti. Milano:

Mursia, 1982.

- Operette morali. A cura di P. Ruffilli. Milano: Garzanti, 1982.

. Pensieri. A cura di C. Galimberti. Milano: Adelphi, 1982.

- Operette morali. Trans. G. Cecchetti. Berkeley: U of California P, 1982.

- Operette morali. Trans. P. Creagh. Manchester: Carcanet, 1983.

- Pensieri, trans. W.S. Di Pietro. Baton Rouge: Louisiana State U P, 1981.

LEVI, G.A. Fra Arimane e Cristo: Leopardi pensatore. Napoli: Istituto Editoriale del 
Mezzogiorno, 1953.

LONARDI, G. Leopardismo: saggio sugli usi di Leopardi dall'Otto al Novecento. Firenze: Sansoni, 1974. $6-49$.

. "Leopardi, Browning e tre poesie di Montale." Studi Novecenteschi I.3 (1972):

MELCHIORI, G. “H. James: Burbank or Bleinstein?" I Henry James e Venezia. Firenze: Olschki, 1986.

NALDINI, N. Leopardi: la vita e le lettere. Con prefazione di F. Bandini. Milano: Garzanti, 1983.

ORIGO, I. Leopardi: a Biography. Oxford: Oxford U P, 1939.

PALMER, D. Shelley: His Reputation and Influence. Doncaster: Metropolitan Inst. of Higher Education, 1983.

PARRONCHI, A. "Il muro di Berkeley e la siepe di Leopardi o la nascita della veduta indiretta." Paragone 114 (giugno 1959).

PERELLA, N.J. Night and the Sublime in G. Leopardi. Berkeley: U of California P, 1970.

PERUZZI, E. Leopardi e i Greci. Firenze: Olschki, 1979.

PRETE, A. Il pensiero poetante. Milano: Feltrinelli, 1980.

PRETE, A. Il demone dell'lanalogia. Milano: Feltrinelli, 1986.

RIGONI, M.A. Saggi sul pensiero leopardiano. Padova: Cluep, 1982.

RIGONI, I. Saggi sul pensiero leopardiano. Napoli: Liguori, 1985.

ROBB, N.E.A. Four in Exile. London: Hutchinson and Co., 1948.

ROGERS, S. Classical Greece and the Poetry of Chénier, Shelley and Leopardi. London: U of Notre Dame P, 1974.

ROSENTHAL, A.S. Baudelaire and Leopardi: A Study in Affinity and Contrasts. Diss. Rutgers, 1970.

SAINTE-BEUVE, C.A. Causerie du Lundi: 24-X-1850.

. Portraits contemporaines. Paris: Calmann Lévy, 1976-79.

SANSONE, M. "Leopardi e la filosofia del Settecento." Leopardi e il Settecento. Firenze: Olschki, 1964.

SANTAYANA, G. Three Philosophical Poets. New York: Scribner's, 1911.

SECRÉTAN, D. Classicism (The Critical Idiom). London: Methuen, 1973.

SHELLEY, P.B. The Complete Poetical Works of P.B. Shelley, ed. T. Hutchinson. London: Oxford U P, 1961.

. Adonais: A Critical Edition. Ed. A.D. Knerr. New York: Columbia U P, 1984.

- La difesa della poesia. A cura di E. Cecchi. Lanciano: Carabba, 1810.

. Il trionfo della vita e altre poesie. A cura di G. Caliumi e R. Sanesi. Milano:

Guanda, 1982.

. Poesie di P.B. Shelley. A cura di R. Sanesi. Milano: Mondadori, 1983.

SCHLEGEL, F. Sämmtiche Werche. Wien, 1823.

SINGH, C. Leopardi e l"Inghilterra. Firenze: Le Monnier, 1968.

STELLA, V. "Sentimento e teoresi in Leopardi: introduzione ad un riesame della sua "estetica.'" Discorsi. V.2 (1985): 253-285.

STUFFERI Malmignati, C. Leopardi nella coscienza dell'Ottocento. Roma: Bonacci, 1976.

TOMALIN, C. Shelley and his World. London: Thames \& Hudson, 1980.

VALENTINI, A. Leopardi. L'io poetante. Roma: Bulzoni, 1983. 
VALLONE, A. Cultura e poesia in Leopardi. Napoli: Liguori, 1981.

WASSERMAN, E.R. Shelley's Prometheus Unbound: A Critical Reading. Baltomore:

J. Hopkins U P, 1965.

WEBB, T. Shelley: a Voice not Understood. Manchester: Manchester U P, 1977.

. The Violet and the Crucible: Shelley and Translalion. Oxford: Clarendon, 1976.

WELLEK, R., and A. Warren. Theory of Literature. London: Cape, 1966.

. Discriminations: further Concepts of Criticism. New Haven: Yale University Press, 1970.

WOODMAN, R.G. The Apocalyptic Vision in the Poetry of Shelley. Toronto: U of Toronto P, 1966. 